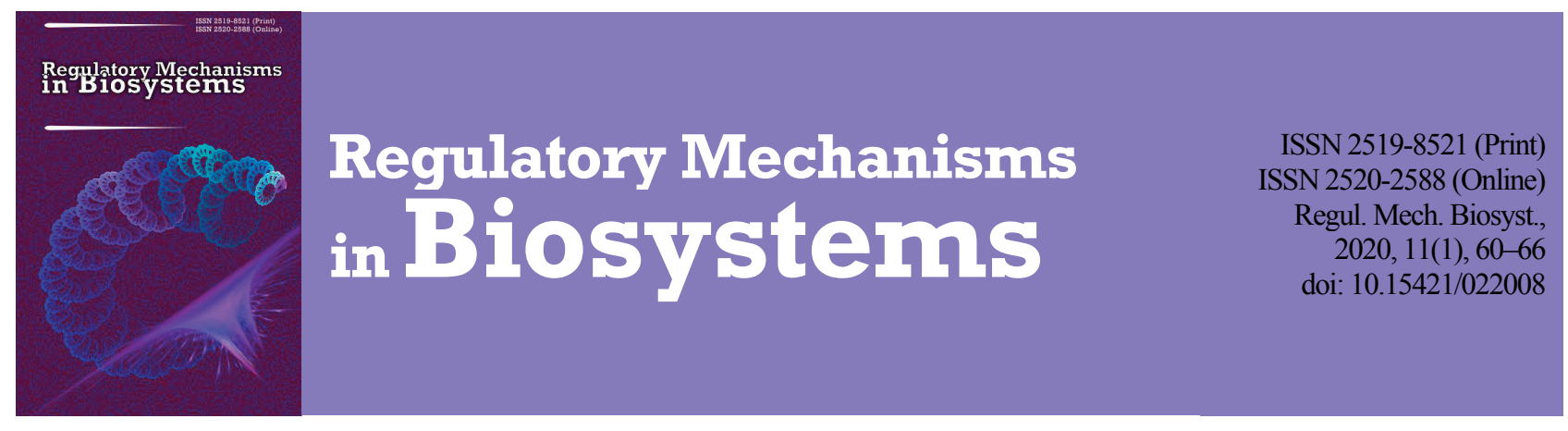

\title{
The effect of pre-sowing seed treatment on seedlings growth rate and their excretory activity
}

\author{
A. I. Bozhkov, M. K. Kovalova, Z. A. Azeez, A. V. Goltvjansky \\ V. N. Karazin Kharkov National University, Kharkov, Ukraine
}

Article info

Received 29.01.2020

Received in revised form 21.02.2020

Accepted 22.02.2020

V. N. Karazin Kharkov National University,

sq. Svobody, 4,

Kharkov, 61022, Ukraine.

Tel. +38-057-707-53-40.

E-mail: bozh-

kov@univer.kharkov.ua
Bozhkov, A. I., Kovalova, M. K., Azeez, Z. A., \& Goltvjansky, A. V. (2020). The effect of pre-sowing seed treatment on seedlings growth rate and their excretory activity. Regulatory Mechanisms in Biosystems, 11(1), 60-66. doi:10.15421/022008

The importance of studying pre-sowing seed treatment lies in the possibility of regulating the rate of seed germination, the intensity of their growth and obtaining root exudates in biotechnology. The effect of three pre-sowing treatment methods was examined (control - washing with running water; the first method - washing with $0.05 \%$ sodium permanganate solution; the second method 30 seconds in $70 \%$ ethyl alcohol $\left(\mathrm{C}_{2} \mathrm{H}_{5} \mathrm{OH}\right)$ and 30 minutes in $5 \%$ sodium hypochlorite $(\mathrm{NaOCl})$; the third method -5 minutes in $70 \% \mathrm{C}_{2} \mathrm{H}_{5} \mathrm{OH}$ and 40 minutes in $5 \% \mathrm{NaOCl}$ ) on the growth rate, germination rate, excretion rate of seeds of wheat and peas and composition (of protein, carbohydrate, amino acid content) of root exudates from the first to the third day of growth in order to obtain root exudates. It was revealed that the same pre-sowing treatment of wheat and pea seeds has a different effect on the rate and variability of seedling growth from the first to the third day, as well as on the qualitative and quantitative composition of root exudates. It was shown that pre-sowing treatment of wheat and pea seeds for 5 minutes with $70 \%$ ethanol followed by treatment with sodium hypochlorite (a "hard" treatment method) accelerates seedling growth and seed germination. This method of treatment reduces the intensity of excretion of root exudates and composition in wheat, but it increases the intensity of excretion in peas. The discovered effects can be explained by hormesis. Additionally, the third method of pre-sowing seed treatment can be used in root technologies for obtaining root exudates.

Keywords: pre-sowing treatment; wheat; peas; root exudates; hormesis; excretion.

\section{Introduction}

The development of biotechnology and bioengineering can significantly increase the productivity of plant objects. Currently, several biotechnological approaches for regulating plant growth and expanding the scope of their metabolites can be distinguished by: 1 - genetic modification; 2 - increase in productivity due to pre-sowing treatment of seeds and the use of growth regulators; and 3 - development of new methods for producing metabolites, including root exudates, 4 - the introduction of rhizospheric microorganisms in agriculture (Król et al., 2014; Majeed et al., 2015; Shen et al., 2019).

The most important point in this regard (pre-sowing treatment) is the use of pre-sowing treatment of seeds and the preparation of root exudates with biological activity (Li et al., 2013; Kuznetsova et al., 2019).

It was shown that the irradiation of seeds before sowing provides an increase in productivity of about 5-20\% (Farooq et al., 2019). Such an increase in yield after irradiation or after the action of other physical factors is explained by the presence of a metabolic acceleration zone on the dose curve and the yield as well. This effect was called radiation hormesis and can be explained by the fact of stress "moderate strength", that is, insufficient for the manifestation of toxicity, provides nonspecific stimulation of metabolism (Kuzin, 1993). For the first time this term was used in 1943 by S. Sontman and D. Erlich and means "fast movement", "aspiration". At the present time it is known that hormesis appears in the culture of microorganisms, plants, and animals, and not only under the influence of radiation, but also other stress factors, including chemical toxicants, that is, it can be attributed to general biological phenomena (Calabrese, 2008; Nweke \& Ogbonna, 2017). Intensive studies of hormesis mechanisms are currently underway. It was shown that the manifestation of hormesis is associated with the induction of stress-proteins (Dattilo et al., 2015), the rearrangement of metabolism, and an increase in the proliferative activity of cells. Knowledge of hormesis mechanisms is crucial in understanding the features of the formation of the response of biological systems to the action of environmental factors and particularly of toxic substances and can be used in practice not only in agriculture but also in medicine. In this regard, further studies of the phenomenon of hormesis are the most important task of biology and biotechnology. The available data suggests that the transition from a stable (normal) metabolic state to a stress state that is induced by negative environmental factors occurs through the formation of unstable metabolic states. It can be argued that an increase in the variability of the functioning of the genome, metabolome, and the formation of phenotypic indicators is a condition for the transition from a relatively stable to a metastable state, and as a result, it manifests itself as hormesis (Tharmalingam et al., 2017).

To test this hypothesis, we studied wheat and pea seedlings in the early stages of growth, from the first to the third day of growth, which went through different methods of pre-sowing seed treatment. That included processing the seeds which used solutions which have a pronounced antibacterial effect such as sodium hypochlorite and alcohol.

This early growth period is interesting because epigenetic patterns are formed in the initial stages that can persist in ontogenesis (Ashapkin et al., 2019). In addition, asynchronous passage of the cell cycle of intensely divided root cells takes place during this period. At the same time, the root rhizosphere and excretory system of the root are formed, which provides a transition to heterotrophic plant nutrition. Also in this early period of growth, the basic metabolic characteristics of plants are formed, which determine their resistance to stress factors, in particular growth intensity and yield (Dupont \& Altenbach, 2003).

An analysis of the functioning of the root excretory system after pre-sowing seed treatment, which provides a hormesis effect in relation 
to the seedling growth rate, plays a vital role in the development of root biotechnologies in order to obtain biologically active compounds (Belz et al., 2011; Kossmann, 2012; Azeez et al., 2018).

In this regard, the influence of different methods of presowing treatment of pea and wheat seeds on germination, the growth rate of seedlings, as well as the intensity of excretion of root exometabolites and their composition from the first to the third day of growth was determined.

\section{Materials and methods}

The objects of the study were the seeds of pea (Pisum sativum) and wheat (Triticum aestivum) of the year 2017. Three methods of pre-sowing treatment of seeds were used (Fig. 1). Control variant: wheat and pea grains were washed 3 times with running water and 3 times with distilled sterile water. The first pre-sowing treatment: wheat, and pea grains after washing, as described in the control version, were soaked in a $0.05 \%$ sodium permanganate solution for 5 minutes, repeatedly (3-4 times) washed with distilled sterile water. The second pre-sowing treatment method: after control wash, wheat and pea grains were soaked for 30 seconds in $70 \%$ ethanol $\left(\mathrm{C}_{2} \mathrm{H}_{5} \mathrm{OH}\right)$, then washed with sterile distilled water and then soaked for 30 minutes in 5\% sodium hypochlorite $(\mathrm{NaOCl})$. After this, the grains were washed with sterile distilled water.

The third method of pre-sowing treatment of seeds differed from the second method by the time of soaking the grains in ethanol and sodium hypochlorite. That is, after control wash, wheat and pea grains were soaked for 5 minutes in $70 \%$ ethanol $\left(\mathrm{C}_{2} \mathrm{H}_{5} \mathrm{OH}\right)$, then washed with sterile distilled water and then soaked for 40 minutes in $5 \%$ sodium hypochlorite $(\mathrm{NaOCl})$. After this, the grains were washed with sterile distilled water.

Indicators
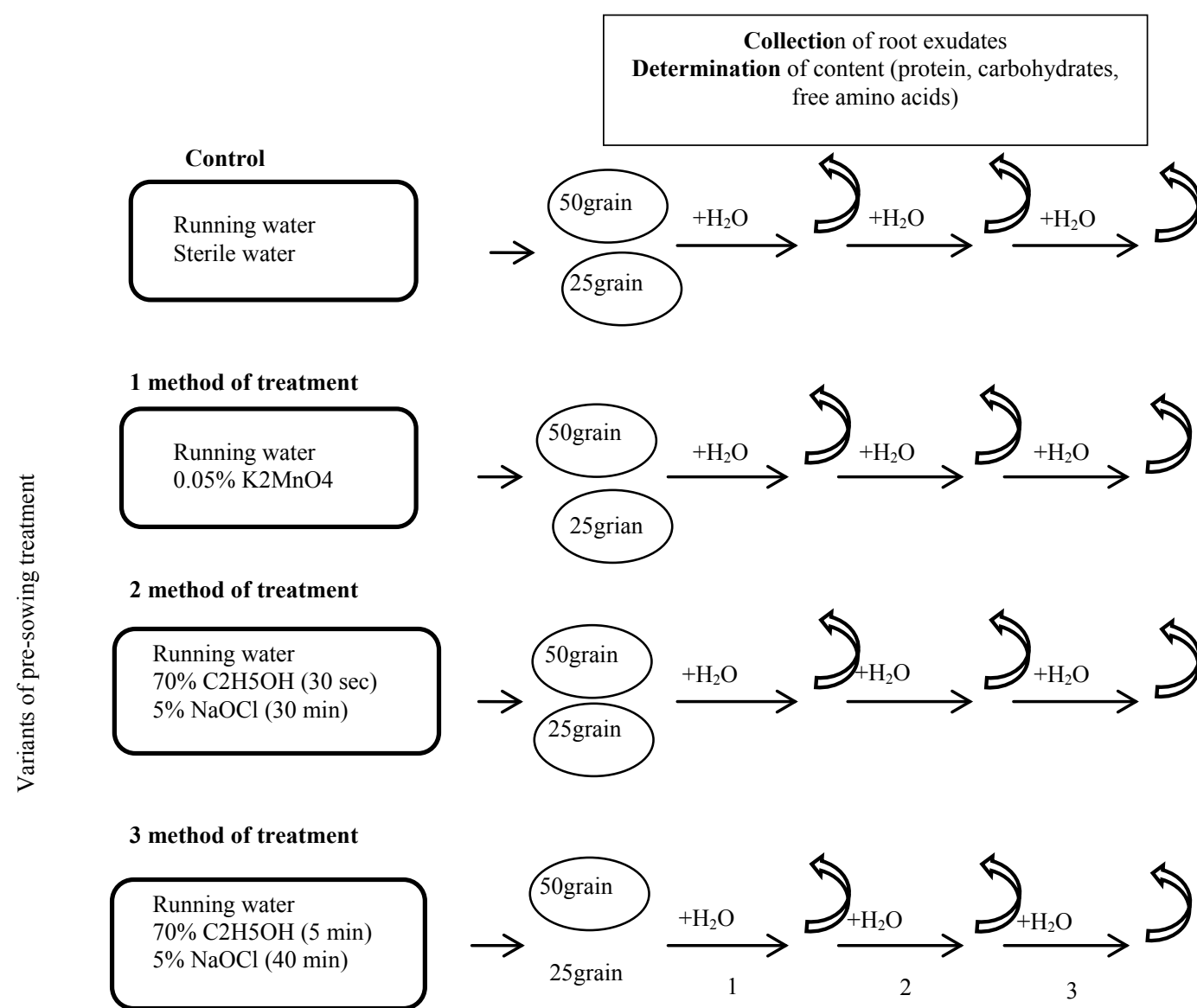

method of treatment
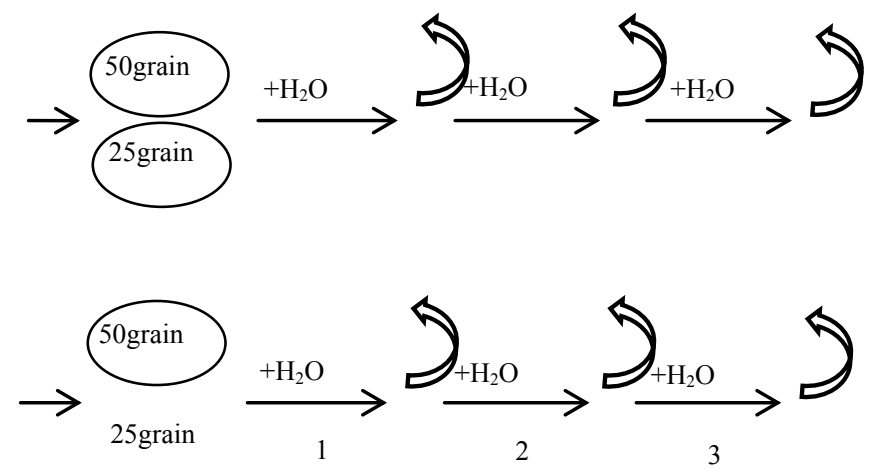

The cultivation time, day

Fig. 1. Experimental design, which demonstrates the sequence of pre-sowing seed treatment, collection of root exudates and determination of protein, carbohydrate, free amino acid content: the figure shows the number of wheat grains (50) and pea grains (25) in every Petri dish; in every experiment, 450 grains of wheat and 225 grains of peas were analyzed; in this work, 3 independent experiments were performed

After pre-sowing treatment, wheat and pea grains were soaked in sterile distilled water for 24 hours at $26^{\circ} \mathrm{C}$ so that the grains would hatch. 24 hours after soaking, the grains from each treatment option were laid out in Petri dishes. 50 grains of wheat were laid out in each Petri dish, and 25 grains of peas. In Petri dishes, distilled water in a volume of 7 and $10 \mathrm{~mL}$, respectively, was added to wheat and pea grains. Dishes with seedlings were placed in a chamber with round-the-clock lighting and a temperature of $26^{\circ} \mathrm{C}$. On the first, second and third days of growth, the number of sprouted grains, the length of the root of pea and wheat seedlings were determined, and aqueous root exudates were collected in a flow mode, in which the content of total proteins, free amino acids and total carbohydrates was determined (Fig. 1).

Determination of the growth rate of wheat and pea seedlings root and the collection of root exudates. To assess the growth rate of the roots of wheat and pea seedlings, the root length of the seedlings from the first to the third day of growth was determined.

To characterize the excretory activity of wheat and pea seedlings, aqueous exometabolites of seedlings were collected in a flow mode. So, an aqueous solution of root exometabolites of one-day old seedlings is exudates that were collected in the first day of growth. Then, to the remaining seedlings of wheat and peas, $7 \mathrm{~mL}$ of distilled sterile water for wheat and $10 \mathrm{~mL}$ for peas was again added to the same Petri dishes and cultivation was repeated under the same conditions for 24 hours. Then, in the second day of growth, an aqueous solution of root exometabolites was collected - these were two-day old root exudates. In the same way, three-day-old root exudates were obtained (Fig. 1). The content of total protein, free amino acids, and total carbohydrates was determined in the first, second, and third day-old root exudates of wheat and pea seedlings. 
The total protein content in the root exudates of wheat and pea seedlings was determined by the Lowry method (Lowry et al., 1951). Protein content was expressed in $\mathrm{mg} / 100$ grains.

The carbohydrate content in the composition of root exudates was determined according to the Molish method (Masuko et al., 2005), and the content of free amino acids was determined using the ninhydrin method as described previously in our works (Azeez et al., 2018). Carbohydrate content was expressed in $\mu \mathrm{g}$ glucose/ 100 grains, and amino acid content in $\mu \mathrm{g}$ glycine/100 grains.

All experiments were carried out in 3 technical replicates (1 Petri dish with 50 wheat seeds each, 1 Petri dish with 25 pea seeds each) and 3 biological replicates. Therefore, 450 seeds of wheat or 225 seeds of pea were used in each treatment group. From each Petri dish with wheat seeds 15 seeds were randomly analyzed, from each Petri dish with peat seeds 20-25 seeds were randomly analyzed. Statistical data analysis was performed using the Statistica 6.0 package (StatSoft Inc. USA, 2001). The data are presented as $\mathrm{x} \pm \mathrm{SE}$ ( $\mathrm{x} \pm$ standard error). The differences between samples were analyzed using the non-parametric MannWhitney U-test (Mann \& Whitney, 1947). Differences were considered statistically significant at $\mathrm{P}<0.05$.

\section{Results}

The influence of different methods of pre-sowing treatment of seeds on the growth rate of wheat and pea seedlings from the first to the third day of growth. It is known that, at the initial stages of germination, intensive root growth is observed in both wheat and peas. So, on the first day the root length of the wheat reached $0.76 \pm 0.03 \mathrm{~cm}$, and length of the peas' root is $0.58 \pm 0.03 \mathrm{~cm}$. On the third day, respectively, $2.78 \mathrm{~cm}$ in wheat and $1.78 \mathrm{~cm}$ in peas (Fig. 2a, c). It should be noted that the growth rate of seedlings from the first to the third day was non-linear for both wheat and peas, with a maximum specific growth rate on the second day of growth $(1.13 \mathrm{~cm} /$ day in wheat roots and $0.79 \mathrm{~cm} /$ day in peas) (Fig. $2 \mathrm{~b}, \mathrm{~d})$.

Pre-sowing treatment of wheat seeds by the first method did not affect the intensity of root growth (Fig. 2a). However, the specific growth rate exceeded the control on the second day of growth, while on the third day, it lagged behind the control (Fig. 2b). Accordingly, pre-sowing treatment of wheat seeds with sodium permanganate increased the variability of the relative root growth rate if compared to the control variant.

In that case, when wheat seeds were treated by the second method $(30 \mathrm{~s}$ with alcohol and $30 \mathrm{~min}$. with sodium hypochlorite) they exceeded the control variant in root length by $25 \%$ on the third day and in specific growth rate in the second and third days (Fig. 2a, b). The greatest stimulating effect on absolute (root length) and specific growth rate of wheat roots was observed after the third method of pre-sowing treatment of wheat seeds (Fig. 2a, b). So, the root length on the third day exceeded the control variant by $40 \%$. The specific growth rate was increased by $36 \%$ on the second day and $98 \%$ on the third day with the comparison of the control variant. It should be noted that the specific growth rate, in the three cases (control variant, the first and the second methods of pre-sowing seed treatment), decreased on the third day if compared with second day, but after the third treatment method it remained the same as on the second day of growth (Fig. 2b). In the event, when pea seeds were subjected to presowing treatment, the first and second methods of pre-sowing seed treatment did not affect the intensity of root growth, while the third treatment method accelerated their root growth (Fig. 2c, d). Thus, the root length exceeded the control variant about $106 \%, 99 \%$, and $103 \%$ on the first, second, and third days, respectively (Fig. 2c). The specific growth rate was also significantly higher than the control values (Fig. 2d).

In the next step, the area of the strange attractor was determined, which depends on the diversity of the trajectories of the dynamics of the studied parameters in the phase space. The area of the strange attractor reflects the variability (instability) of the behaviour dynamics of research indicators. Thus, the phase space area, constructed from changes in the trajectories of wheat root growth dynamics in the control variant from the first to the third day, increased from the first to the second day about 7 times and 3.5 times from the second to the third day of growth (Fig. 3a).

Pre-sowing treatment of wheat seeds was accompanied with an increase in the "strange attractor" area on the first day of the seedlings growth of about two times compared with the control regardless of the method of pre-sowing treatment seeds (Fig. 3a). But on the second and third days, they did not significantly differ from the control (Fig. 3a). Therefore, the variability of the growth dynamics of wheat roots in the control variant increased repeatedly from the first to the third day of growth. Pre-sowing treatment of wheat seeds increased the variability of growth dynamics only on the first day of wheat roots and this did not depend on the method of pre-sowing treatment.

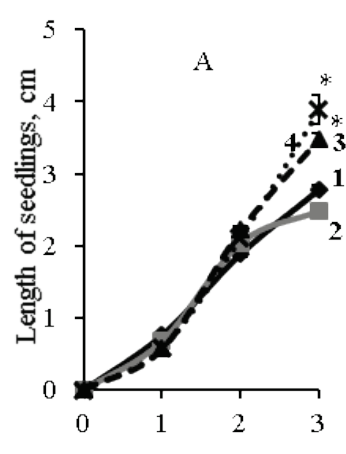

Days of culture

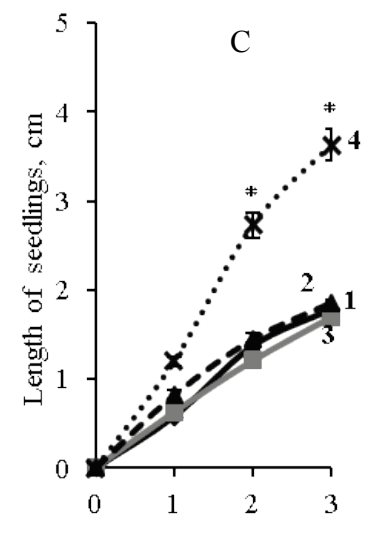

Days of culture

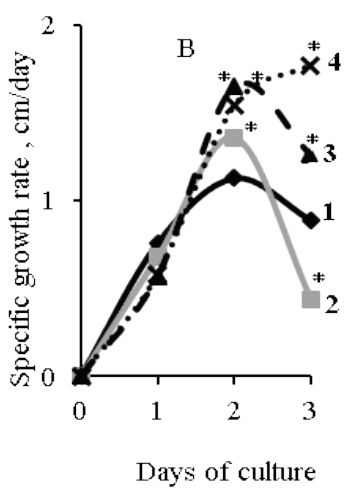

D

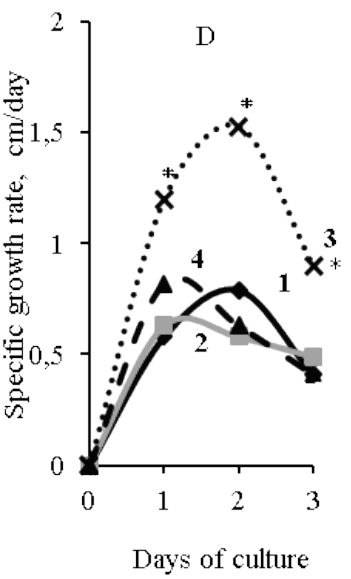

Fig. 2. The length of wheat roots $(a)$ and the specific growth rate (b); the length of pea roots $(c)$ and the specific growth rate $(d)$ : in the control version ( $(1)$ and after pre-sowing treatment: 2 - treatment with potassium permanganate, 3 - with ethyl alcohol and sodium hypochlorite (alcohol for 30 secs, sodium hypochlorite for $30 \mathrm{~min}$ ); 4 - with ethyl alcohol (5 min) and sodium hypochlorite (40 min); the quantity of wheat seeds was $n=135$, the quantityof pea seeds was $n=180$ in each treatment group; the data are presented as $x \pm$ $\mathrm{SE}(\mathrm{x} \pm$ standard error); the differences between samples were analyzed using the non-parametric Mann-Whitney U-test; differences were considered statistically significant at $\mathrm{P}<0.05$; ${ }^{*}-\mathrm{P}<0.05$ compared with the control

The strange attractors for the growth dynamics of pea roots were very different from the strange attractors of wheat roots (Fig. 3b). So, in oneday-old pea roots, the area of the strange attractor was two times bigger than the attractor area of wheat in the control variant. The attractor area did not significantly change from the first to the third day of growth. Presowing treatment of pea seeds did not affect the area of strange attractors on the second and third days of growth (Fig. 3b).

Accordingly, the use of strange attractors allows us to identify differences in the nonlinear dynamics of changes in the root growth indicators and it can be used in assessing the variability of biological indicators.

Such variability of the dynamics of root growth may be due to different germinations of seeds.

\section{The effect of pre-sowing seed treatment on germination}

In the control variant of wheat seeds, at the end of the first day of growth, $26 \%$ of the grains remained non-germinated, while at the end of 
the second day their number had decreased and $14 \%$ grains remained non-germinated, and at the end of the third day $6 \%$ of the seeds were nongerminated (Fig. 4a). As a result, the wheat seeds of the control variant were quite heterogeneous in terms of germination rate. This was manifested in a linear decrease in the number of non-germinated seeds from the first to the third day of growth (Fig. 4a).
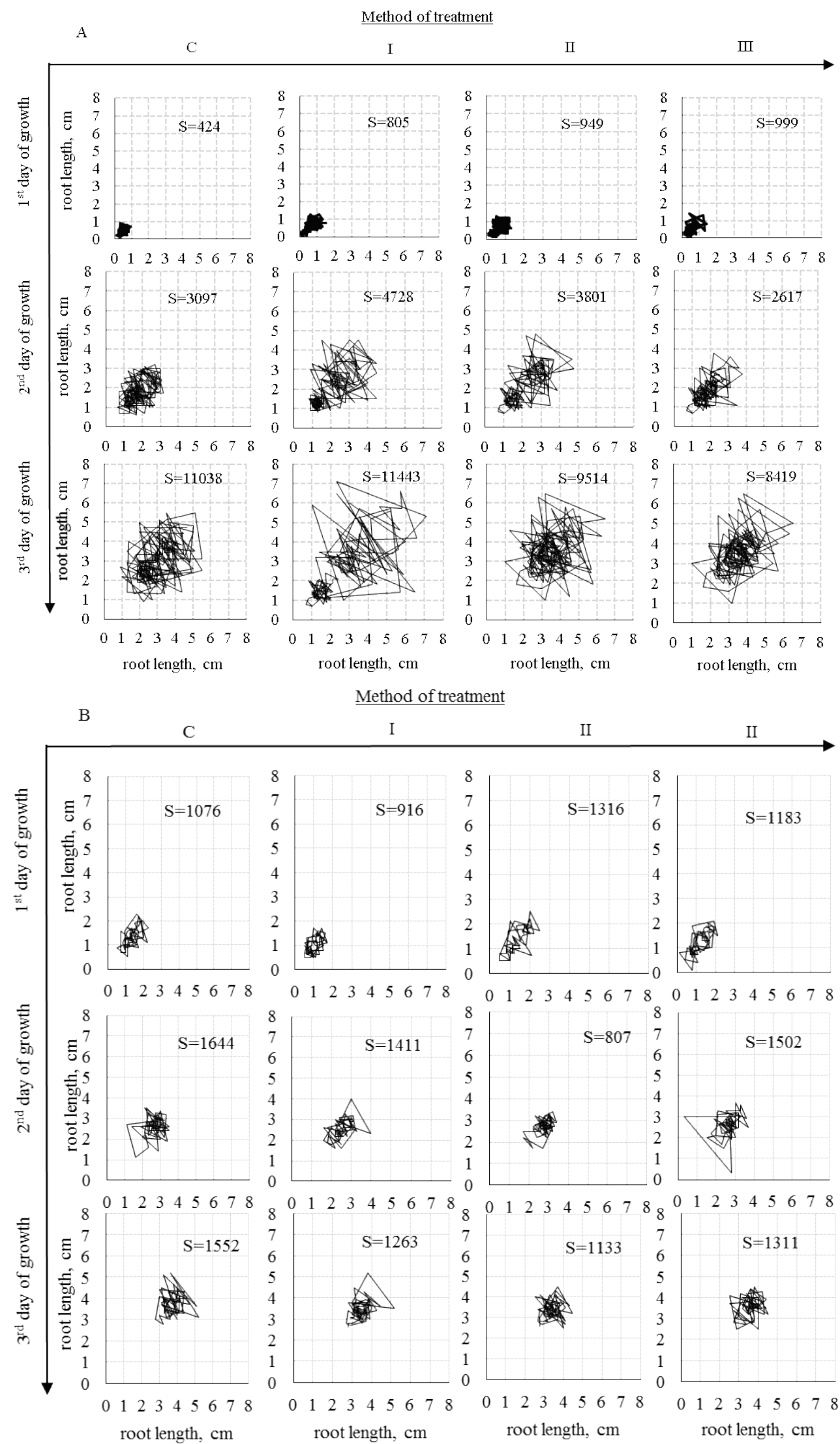

Fig. 3. Attractors for the root length of wheat seedlings from 1st to 3rd day of growth: wheat grains $(a)$ and pea grains $(b)$ were processed in different ways: $c$ - control variant, $I$ - pre-sowing treatment - potassium permanganate, $I I$ - ethyl alcohol ( $30 \mathrm{~s})$ and sodium hypochlorite (30 min); III - ethyl alcohol ( $5 \mathrm{~min}$ ) and sodium hypochlorite $(40 \mathrm{~min})$; the strange attractors for root length were built using the time delay method with Statistica 6.0 software package; the quantity of wheat seeds were $n=135$, the quantity of pea seeds were $n=180$ in each treatment group 

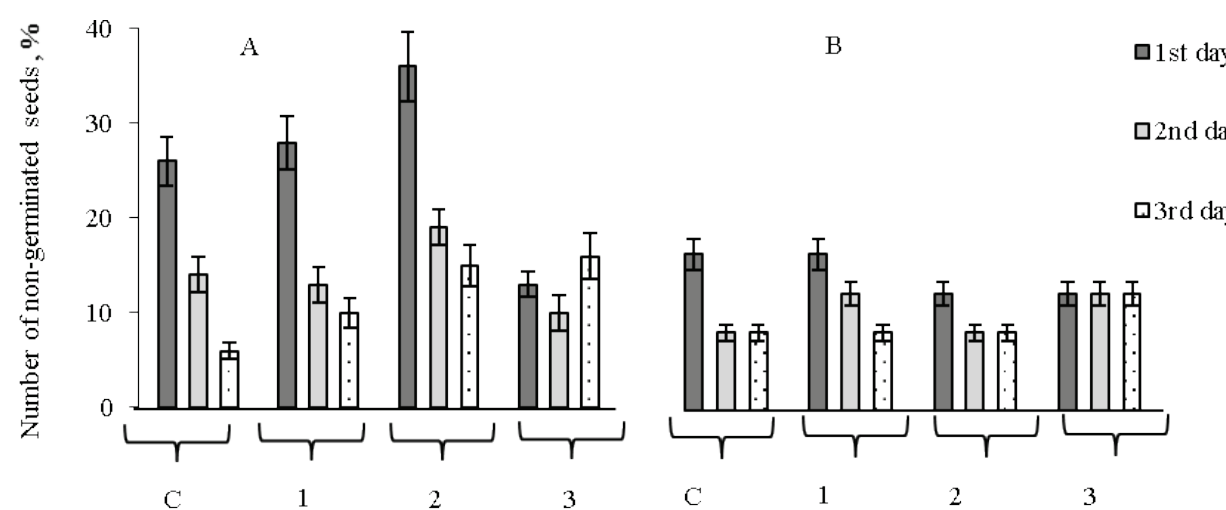

Fig. 4. The number of non-germinated seeds of wheat $(a)$ and pea $(b)$ in the control variant $(c)$ and after pre-sowing treatment: 1 - treatment with potassium permanganate, 2 - treatment with ethyl alcohol and sodium hypochlorite (alcohol for $30 \mathrm{~s}$, sodium hypochlorite for $30 \mathrm{~min}) ; 3$ - treatment with ethyl alcohol (5 $\mathrm{min}$ ) and sodium hypochlorite (40 $\mathrm{min})$; $\mathrm{x} \pm \mathrm{SE}$; the differences between samples were analyzed using the non-parametric Mann-Whitney U-test $(\mathrm{P}<0.05)$

When the wheat seeds were treated by the first method, the number of non-germinated seeds did not differ from the control variant (Fig. 4a). However, when wheat seeds were treated by the second method, then the number of non-germinated seeds at the end of the first day was $36 \%$, and on the third day $15 \%$ versus $6 \%$ in the control (Fig. 4a). After using the third method of pre-sowing treatment, the number of non-germinated grains was $13 \%$ and it did not change from the first to the third day of growth. Therefore, this method of seed treatment increased the germination of wheat seeds. The germination of pea seeds in the control variant was significantly better than with wheat. So, at the end of the first day of growth non-germinated seeds were only $16 \%$, and after the second and the third days were $8 \%$ of the total (Fig. $4 b$ ). Pre-sowing treatment of pea seeds did not have a significant effect on germination, with the exception of the third method of treatment. After using the third treatment method, the number of non-germinated seeds on the first day was $12 \%$, they did not germinate even on the third day of growth (Fig. 4b). Therefore, the third method of pre-sowing treatment accelerated the germination of pea seeds by about $4 \%$ on the first day if compared with the control variant.

It can be concluded that the same type of pre-sowing treatment of pea and wheat seeds has different effects on seed germination. This could have a special effect on the growth rate of wheat and pea roots. One of the important factors of plant growth is the functional activity of the root system, more precisely, the formation of the microenvironment of the roots. In the process of formation of the microenvironment of the root, root exudates play a decisive role. At the next stage of work, the excretory activity of wheat and peas roots was determined after various methods of pre-sowing seed treatment.

The effect of pre-sowing treatment of wheat and pea seeds on the qualitative and quantitative composition of root exudates

It was found that pre-sowing treatment of wheat seeds influenced the intensity of excretion and this depended on the method of pre-sowing treatment (Fig. 5a). In the control variant, the excretion intensity increased from the first to the second day by about 1.9 times, and from second to the third day by 2.1 times, that is, it almost linearly increased from the first to the third day of growth (Fig. 5a). In this case, when the wheat seeds were treated by the first method, the number of root exudates on the third day of growth was $25 \%$ higher than in the control variant, and after using the second method, it was $19 \%$ more than with the control variant (Fig. 5a). When the seeds were treated by the third method, by contrast, on the third day they excreted only $2,174.9 \mu \mathrm{g} / 100$ grains, but in the control the total number of root exudates was $5,880 \mu \mathrm{g} / 100$ grains, that is 2.7 times less than the total number of root exudates in the control variant (Fig. 5a). Presowing treatment had a completely different effect on the excretory activity of pea seedlings. Firstly, pea seedlings are characterized by other dynamics of excretory activity if compared with wheat seedlings (Fig. 5b). So, at the end of the first day of growth in the medium, $5,833.7 \mu \mathrm{g} / 100$ grains of root exudates were released, exactly as much as was released in the medium by wheat seedlings on the third day of growth. Secondly, on the second day of growth, the number of exudates in peas did not differ from their number on the first day (Fig. 5b). The content of pea exudates on the third day of growth increased only by $22 \%$ if compared with the first and the second days of growth (Fig. 5b). Thirdly, the pre-sowing treatment of pea seeds by the first and second methods did not affect either the dynamics of growth or the number of excreted exudates (Fig. 5). Fourthly, the third method of pre-sowing seed treatment of pea seeds did not inhibit the excretory activity as in wheat but stimulated it without changing the dynamics of this process. So, on the third day, the total number of root exudates was $34 \%$ more than the total number of exudates in the control variant and amounted to almost 9,517.7 $\mu \mathrm{g} / 100$ seeds (Fig. $5 \mathrm{~b}$ ).

Therefore, pre-sowing treatment of seeds had a different effect on the intensity of excretion of root exudates and this depended on both the treatment methods and the species of plant. Of potential interest is the qualitative composition of the root exudates because their biological activity depends on it. The main component of root exudates of wheat (50$70 \%$ of all components) was carbohydrates. Their content practically did not change from the first to third day of seedling growth in the control variant (Fig. 6). Pre-sowing treatment of wheat seeds with the first and second methods did not affect the carbohydrate content of exudates (Fig. 6). However, the third method of pre-sowing treatment of wheat seeds had an impact on the composition of root exudates of wheat. Thus, the content of carbohydrates in the composition of root exudates decreased about 3 times on the first day of growth and 5 times on the third day if compared with the control variant (Fig. 6). Proteins in the composition of root exudates of wheat accounted for $15-25 \%$ of exudates. Their number from the first to the third day of growth also remained unchanged, and the pre-sowing treatment of wheat seeds by the first and second methods did not affect their quantity (Fig. 6). At the same time, after the third method of pre-sowing treatment of wheat seeds, the relative protein content was increased about 3.0-3.5 times in comparison with the control variant (Fig. 6). Of great interest is the content of free amino acids in the composition of root exudates; they accounted for $6-30 \%$ of exudates. The dynamics of their content in wheat exudates were unusual and can be defined as Ushaped. So, their number on the first day was $9 \%$, on the second day of growth it increased to $25 \%$, and on the third day it decreased to $19 \%$ of the total content of exudates. Pre-sowing treatment of wheat seeds with the first and second methods did not affect the number and dynamics of free amino acids in the composition of wheat root exudates (Fig. 6). At the same time, the third method of pre-sowing treatment of wheat seeds significantly reduced the content of free amino acids in the composition of exudates. So, their amount remained the same as on the first day and was 3 times less on the second day and 3 times on the third day of growth if compared with the control variant (Fig. 6). The pea root exudates were very different in composition from wheat root exudates. More than $80 \%$ were proteins, carbohydrates accounted for $10-15 \%$ and $4-6 \%$ for free amino acids. The ratio of these components remained constant from the first to the third day of growth (Fig. 7). Pre-sowing treatment of pea seeds did not affect the composition of root exudates (Fig. 7). Consequently, the excretory activity of pea roots was formed during the first days of growth, pre-sowing treatment of pea seeds did not affect the composition of root exudates, and at least $80 \%$ of the exudates included proteins. 

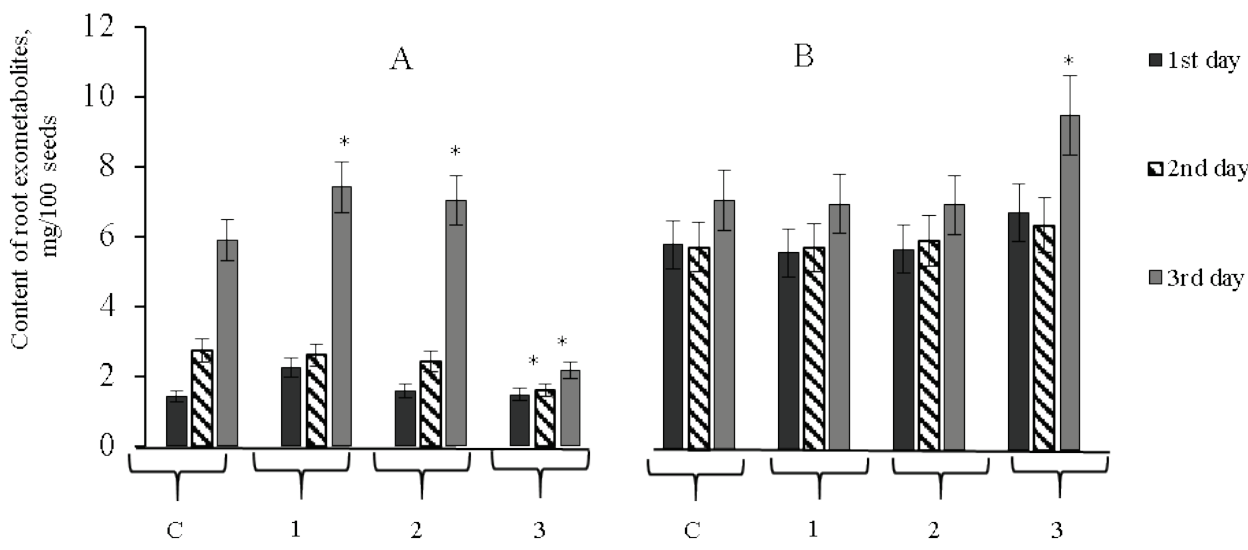

Fig. 5. The content of the total number of root exudates in the aquatic culture of wheat seedlings (a) and peas (b) in the control variant (c) and after pre-sowing treatment: 1 - treatment with potassium permanganate, 2 - with ethyl alcohol and sodium hypochlorite (alcohol for $30 \mathrm{~s}$, sodium hypochlorite for $30 \mathrm{~min}$ ); 3 - with ethyl alcohol (5 min) and sodium hypochlorite (40 $\mathrm{min})$; $\mathrm{x} \pm \mathrm{SE}$; the differences between samples were analyzed using the non-parametric Mann-Whitney U-test: * $-\mathrm{P}<0.05$ compared with the control
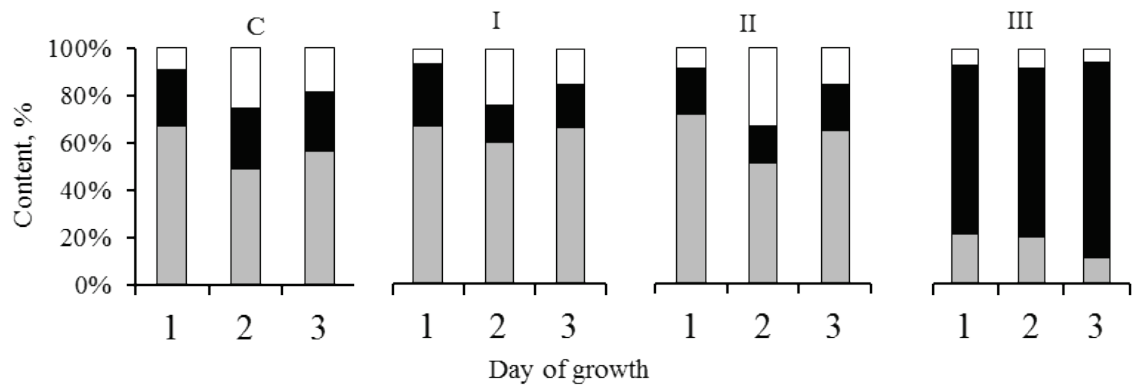

$\square$ amino acid

- protein

$\square$ carbohydrate

Fig. 6. The content (\%) of carbohydrates, total proteins and free amino acids in aqueous root exudates of wheat seedlings, expressed as a percentage of the total number of root exudates: $C$ - control variant, after pre-sowing treatment: $I$ - treatment with potassium permanganate,

$I I$ - with ethyl alcohol and sodium hypochlorite (alcohol for $30 \mathrm{~s}$, sodium hypochlorite for $30 \mathrm{~min}$ ); III - with ethyl alcohol (5 min) and sodium hypochlorite (40 $\mathrm{min})$
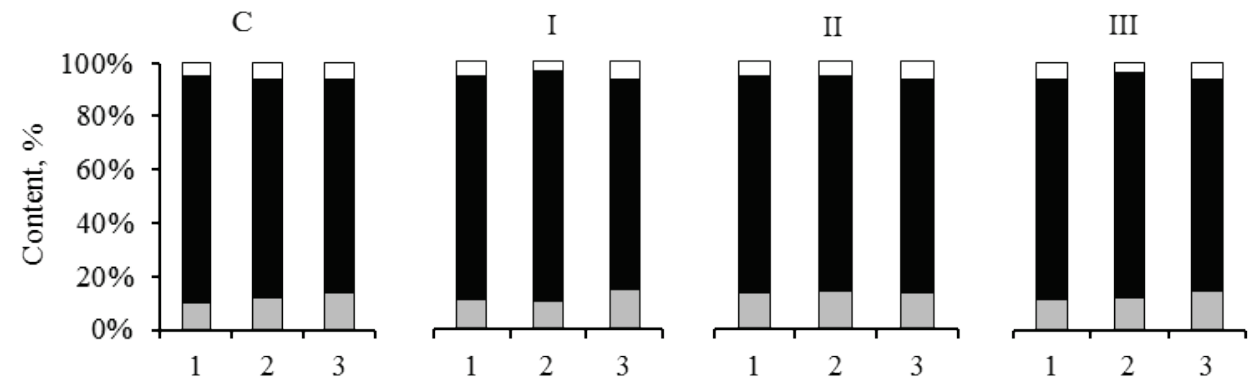

$\square$ amino acid

- protein

$\square$ carbohydrate

Day of growth

Fig. 7. The content of carbohydrates, total proteins and free amino acids in aqueous root exudates of pea seedlings, expressed as a percentage of the total number of root exudates: $C$ - control variant, after pre-sowing treatment; $I$ - treatment with potassium permanganate;

$I I$ - with ethyl alcohol and sodium hypochlorite (alcohol for $30 \mathrm{~s}$, sodium hypochlorite for $30 \mathrm{~min}$ ); III - with ethyl alcohol (5 min) and sodium hypochlorite (40 $\mathrm{min})$

\section{Discussion}

Seed germination is a complex biochemical process that can be characterized as a transition from a state of suspended animation to an active metabolism. This early stage is divided into 5 phases: water absorption, swelling, growth of primary roots, sprout development and seedling formation. The early stage of the formation of growth processes has a decisive influence on further growth and yield formation. Pre-sowing seed treatment, as a rule, should provide increased germination, germination energy and removal of phytopathogens. This is relevant when obtaining root exudates in aquatic cultures in modern biotechnology (Azeez et al., 2018; Wolny et al., 2018; Lechowska et al., 2019). Removal of epiphytic microorganisms is a rather difficult task. It was previously shown that the use of pre-sowing seed treatment can provide partial or complete removal of epiphytic microorganisms (Azeez et al., 2019). It is possible to say that the significant thing to be mentioned is the data of the stimulating effect on the growth rate of wheat and pea seedlings after sequential treatment with sodium hypochlorite and ethyl alcohol because it affects the qualitative and quantitative characteristics of the root excretory system. The various effects of pre-sowing treatment on wheat and pea seeds can be explained by the differences in the structure of seeds and the peculiarities of wheat and pea cell metabolism, which was manifested in the fact that most of the root exudates of wheat are represented by carbohydrates, while the peas represented by proteins. Therefore, using wheat and peas makes it possible to obtain biologically active compounds of different compositions with different biological activity.

The stimulating effect of pre-sowing treatment of seeds can be explained by the fact that sodium hypochlorite and ethyl alcohol can be used in specific concentrations and time intervals. This causes insignificant damage to the surface of seeds and embryonic cells as well. Such a process accelerates the rate of water entry into tissues and increases the proliferative activity of cells as a response to minor damage to cellular compo- 
nents. Generally, all that happens causes a hormesis effect. A similar hormesis effect is shown after exposure to various types of radiation and the action of other physical factors (Calabrese \& Baldwin, 1999; Flores \& Garzon, 2013; Garzon \& Flores, 2013).

The results of this work showed that, firstly, pre-sowing treatment of wheat slightly accelerated the rate of root growth, and this effect is dependent on the type of pre-sowing seed treatment. Secondly, pre-sowing treatment of pea seeds had a different effect on the root growth rate (i.e the first and second methods did not affect the root growth rate of pea seedlings, but the third pre-sowing seed treatment increased the root growth rate about two times). Thirdly, the growth rate of seedlings from the first to the third day was non-linear (the highest specific growth rate was on the second day of growth, and on the third day it decreased), which was typical for wheat seeds and pea seeds. This nature of the nonlinear dynamics of the growth of seedlings and the great diversity of results obtained in biological experiments reflects the fact that biological systems are nonlinear dynamical systems which are characterized by "chaotic" behaviour. To characterize such systems, they use the construction of the dynamics of the behaviour of the system in phase space. A graphic representation of the behaviour system in the phase space is called "attractors". The attractor is characterized by a compact subset of the phase space of a dynamic system, all trajectories from a certain neighborhood of which tend to phase space with time. Such attractors belong to regular attractors. In the case when there is no fixed attracting point, then such an attractor is called a "strange attractor" (Mosekilde et al., 1988; Hastings et al., 1993). Strange attractors have a non-periodic trajectory, an unstable mode of operation is characterized by high sensitivity to the initial conditions.

The study of the relationship between growth processes and the qualitative and quantitative composition of root exudates is important to understand the mechanisms of autoregulation of growth and development of plants, and to obtain root exudates that are used in biotechnology (Bozhkov et al., 1996; Kossmann, 2012; Li et al., 2013; Azeez et al., 2018).

As is known, the main amount of root exudates is composed of sugars, proteins and free amino acids (Canarini et al., 2019). It was shown, that the ratio between the main components of exudates depends not only on cultivation conditions, but also on the biological characteristics of plants and on the species composition of plants (Li et al., 2013).

\section{Conclusion}

Presowing treatment of wheat and pea seeds with ethyl alcohol followed by treatment with sodium hypochlorite stimulated the growth of seedlings from the first to the third day of growth. The stimulated effect was different for wheat and peas. Such stimulation was accompanied by the increased excretory activity of root exudates. Pre-sowing treatment of wheat seeds was accompanied by an increase in the variability of the growth rate of seedlings and did not affect this indicator for pea seedlings. The construction of strange attractors for nonlinear dynamical systems, which are biological systems, can be used to characterize the growth of seedlings in extreme conditions.

\section{References}

Ashapkin, V., Kutueva, L., Aleksandrushkina, N., \& Vanyushin, B. (2019). Epigenetic regulation of plant gametophyte development. International Journal of Molecular Sciences, 20, 3051.

Azeez, Z. A., Bozhkov, A. I., Mahmood, M. T., \& Kovalova, M. K. (2019). The species composition of epiphytic microorganisms and their influence on roots excretory activity of wheat and pea seedlings. Biochemical and Cellular Archives, 19(2), 3809-3818.

Azeez, Z., Kovalova, M., \& Bozhkov, A. (2018). Effects of pre-sowing seed treatment on the growth rate of seedlings and the activity of the excretory system of the wheat root in aquatic culture. International Journal of Agriculture, Environment and Biotechnology, 11(3), 573-583.

Belz, R. G., Cedergreen, N., \& Duke, S. O. (2011). Herbicide hormesis - can it be useful in crop production? Weed Research, 51, 321-332.

Bozhkov, A. I., Menzyanova, N. G., \& Leontovich, V. P. (1996). Lipid composition and antibacterial activity of root exometabolites of wheat seedlings. Russian Journal of Plant Physiology, 43(6), 920-925.
Calabrese, E. (2008). Hormesis: Why it is important to toxicology and toxicologists. Environmental Toxicology and Chemistry, 27(7), 1451-1474.

Calabrese, E. J., \& Baldwin, L. A. (1999). Chemical hormesis: Its historical foundations as a biological hypothesis. Toxicologic Pathology, 27(2), 195-216.

Canarini, A., Kaiser, C., Merchant, A., Richter, A., \& Wanek, W. (2019). Root exudation of primary metabolites: Mechanisms and their roles in plant responses to environmental stimuli. Frontiers in Plant Science, 10, 157.

Dattilo, S., Mancuso, C., Koverech, G., Di Mauro, P., Ontario, M. L., Petralia, C. C., Petralia, A., Maiolino, L., Serra, A., Calabrese, E. J., \& Calabrese, V. (2015). Heat shock proteins and hormesis in the diagnosis and treatment of neurodegenerative diseases. Immun Ageing, 12, 20.

Dupont, F. M., \& Altenbach, S. B. (2003). Molecular and biochemical impacts of environmental factors on wheat grain development and protein synthesis. Journal of Cereal Science, 38(2), 133-146.

Farooq, N., Abbas, T., Tanveer, A., Javaid, M., Ali, H., Safdar, M., Khan, A., Zohaib, A., \& Shahzad, B. (2019). Differential hormetic response of fenoxaprop-p-ethyl resistant and susceptible phalaris minor populations: A potential factor in resistance evolution. Planta Daninha, 37, e019187554.

Flores, F. J., \& Garzon, C. D. (2013). Detection and assessment of chemical hormesis on the radial growth in vitro of oomycetes and fungal plant pathogens. DoseResponse, 11(3), 361-373.

Hastings, A., Hom, C. L., Ellner, S., Turchin, P., \& Godfray, H. C. (1993). Chaos in ecology: Is mother nature a strange attractor? Annual Review of Ecology and Systematics, 24, 1-33.

Kossmann, J. (2012). Grand challenges in plant biotechnology. Frontiers in Plant Science, 3,61 .

Król, P., Adamska, J., \& Kępczyńska, E. (2014). Enhancement of Festuca rubra L. germination and seedling growth by seed treatment with pathogenic Agrobacterium rhizogenes. Acta Physiologiae Plantarum, 36, 3263-3274.

Kuzin, A. M. (1993). The key mechanisms of radiation hormesis. Izvestiya Akademii Nauk Seriya Biologicheskaya, 6, 824-832.

Kuznetsova, Y., Bozhkov, A., Menzyanova, N., Goltvyansky, A., \& Azeez, Z. (2019). Root exudates of wheat seedling express antibacterial and antioxidant activity and stimulate proliferation of liver cells. Indian Journal of Natural Products and Resources, 9(4), 303-310.

Lechowska, K., Kubala, S., Wojtyla, L., Nowaczyk, G., Quinet, M., Lutts, S., \& Garnczarska, M. (2019). New insight on water status in germinating Brassica napus seeds in relation to priming-improved germination. International Journal of Molecular Sciences, 20(3), 540.

Li, X., Zhang, T., Wang, X., Hua, K., Zhao, L., \& Han, Z. (2013). The composition of root exudates from two different resistant peanut cultivars and their effects on the growth of soil-borne pathogen. International Journal Biological Sciences, 9(2), 164-173.

Lowry, O. H., Rosebrough, N. J., Farr, A. L., \& Randall, B. J. (1951). Protein mesurement with folin phenol reagent. The Journal of Biological Chemistry, 193(1), 265-275.

Majeed, A., Abbasi, M., Hameed, S., Imran, A., \& Rahim, N. (2015). Isolation and characterization of plant growth-promoting rhizobacteria from wheat rhizosphere and their effect on plant growth promotion. Frontiers in Microbiology, 6, 198.

Mann, H. B., \& Whitney, D. R. (1947). On a test of whether one of two random variables is stochastically larger than the other. The Annals of Mathematical Statistics, 18, 50-60.

Masuko, T., Minami, A., Iwasaki, N., Majima, T., Nishimura, S. I., \& Lee, Y. C. (2005). Carbohydrate analysis by a phenol-sulfuric acid method in microplate format. Analytical Biochemistry, 339, 69-72.

Mosekilde, E., Aracil, J., \& Allen, P. M. (1988). Instabilities and chaos in nonlinear dynamic systems. System Dynamics Review, 4, 14-55.

Nweke, C., \& Ogbonna, C. (2017). Statistical models for biphasic dose-response relationships (hormesis) in toxicological studies. Ecotoxicology Environmental Contamination, 12(1), 39-55.

Shen, B., Wang, L., Lin, X., Yao, Z., Xu, H., Zhu, G., Teng, H., Cui, L., Liu, E., Zhang, J., He, Z., \& Peng, X. (2019). Engineering a new chloroplastic photorespiratory bypass to increase photosynthetic efficiency and productivity in rice. Molecular Plant, 12, 199-214.

Tharmalingam, S., Sreetharan, S., Kulesza, A. V., Boreham, D. R., \& Tai, T. C. (2017). Low-dose ionizing radiation exposure, oxidative stress and epigenetic programing of health and disease. Radiation Research, 188, 525-538.

Wolny, E., Betekhtin, A., Rojek, M., Braszewska-Zalewska, A., Lusinska, J., \& Hasterok, R. (2018). Germination and the early stages of seedling development in Brachypodium distachyon. International Journal of Molecular Sciences, 19(10), 2916. 